\title{
INVENTORY CONTROL OF SPARE PARTS USING A BAYESIAN APPROACH: A CASE STUDY
}

\author{
Econometric Institute Report EI-9950/A \\ Kostas-Platon Aronis ${ }^{1}$, Ioulia Magou ${ }^{1}$, Rommert Dekker ${ }^{2}$, George Tagaras ${ }^{1}$ \\ ${ }^{1}$ Aristoteles University of Thessaloniki \\ ${ }^{2}$ Erasmus University Rotterdam
}

\begin{abstract}
This paper presents a case study of applying a Bayesian approach to forecast demand and subsequently determine the appropriate parameter $S$ of an $(S-1, S)$ inventory system for controlling spare parts of electronic equipment. First, the problem and the current policy are described. Then, the basic elements of the Bayesian approach are introduced and the procedure for calculating the appropriate parameter $S$ is illustrated. Finally, we present the results of applying the Bayesian approach in an innovative way to determine the stock levels of three types of circuit packs at several locations. According to the proposed method, a lower base stock than the one currently used is sufficient to achieve the desired service level.
\end{abstract}

Keywords : inventory control, spare parts, Bayesian analysis, case study

- Correspondence: Prof. R. Dekker, Econometric Institute, Erasmus University Rotterdam, The Netherlands, Email: rdekker@few.eur.nl 


\section{INVENTORY CONTROL OF SPARE PARTS USING A BAYESIAN APPROACH: A CASE STUDY}

\section{Introduction}

Inventory control of spare parts plays an increasingly important role in modern operations management. The trade-off is clear: on one hand a large amount of spare parts ties up a large amount of capital, while on the other hand too little inventory may result in poor customer service or extremely costly emergency actions. This paper studies a specific case, where a company producing circuit packs as spare parts for telephone switching systems has a policy of keeping its customers content by maintaining a sufficiently high service level.

The company, which for confidentiality purposes will be called by the fictitious name "Katharo Technik", KT for brevity, designs, develops and manufactures communication systems. One class of its products is circuit packs with specialised software downloaded in them. These circuit packs are parts of electronic equipment, installed in telephone switching systems at large communication firms. It is clear that continuous operation of these systems is essential for KT customers. When such a system ceases to operate because of a failure in a circuit pack, it has to be immediately restored by replacing the failed circuit pack with a readily available spare part. Thus, the demand for spare parts originates by the random failures of the installed circuit packs.

Since the availability of a suitable spare part at the moment it is required is crucial, the customers buy a priori the spare parts they need and keep them in KT's warehouses. That means all circuit packs in the warehouses are property of the customers. There is a central KT warehouse and local warehouses in several locations, in close proximity to the main customers. Circuit packs are distinguished as service affecting and non-service affecting. Failure of the former has serious consequences on the availability of a switch to the end user and consequently these parts are stocked locally to reduce downtime. Non-service affecting circuit packs are stocked centrally. When a failure of a circuit pack in a switch is observed, a spare part is retrieved from a KT warehouse and serves as a replacement. The replacement is performed by maintenance personnel. The entire procedure lasts four hours when the spare part comes from central stock and only two hours if it comes from a local warehouse. The defective circuit pack is transported to the KT repair centre. After repair, the circuit pack is usually restored to as good as new condition and it is put back in the central or local 
warehouse. If it is beyond repair to a satisfactory condition, it is replaced in stock by a new one.

KT comes in agreement with its customers on the service level it has to provide them and then tries to determine the minimum stock level that satisfies the customer requirements at each stocking location separately, taking into account the respective circuit packs in use. KT sees the spare parts stock as an insurance against unexpected events. In this regard there is uncertainty both with respect to the average failure rate and with respect to the usual fluctuations given an average failure rate. KT's present method tackles both types of uncertainty separately. The Bayesian method we propose tackles them in an integral way and gives a better indication of which service level one may finally expect. Although the Bayesian method is not new in inventory control (see e.g. Kaplan ${ }^{1}$ and Sherbrooke ${ }^{2}$ ), the application is.

The next section describes current practice for stock level calculation at KT. The challenge and motivation behind this case study was to improve the current method without altering the type of the inventory control policy. The vehicle to this end is a more accurate forecast of the demand for spare parts, based not only on using all available past information, but also on being "sensitive" to the new data observed each time a failure occurs. The following section presents the main elements of a Bayesian approach, which is appealing because of its updating capability. As the choice of initial prior distribution parameters is a key but subjective element of the Bayesian approach, this section also contains a discussion and sensitivity analysis on the values of these parameters. Thereafter we present the actual application of a suitably adjusted variation of the Bayesian method to the inventory control of specific circuit packs and compare the results with those under the current method of stock level determination.

\section{Current practice}

KT has established that the $(S-1, S)$ inventory control model shall be used for managing the inventory of spare parts. Consequently, the specification of the inventory control policy entails the calculation of the base stock level $S$ that fulfils customer requirements at each particular case. Currently, the calculation is based on the assumption that the time to failure of a circuit pack follows an exponential distribution (a valid assumption given the random nature of electronic equipment failures) with parameter ?, which is constant but different from one type of circuit pack to another. Therefore, the 
number of failures (demand for spare parts) of a circuit pack type during the replenishment order lead-time $L$ follows a Poisson distribution with mean ?nL, where $n$ is the number of installed circuit packs of that type. The company calculates $S$ for a given service level $p$ (probability that a demand for a spare part is immediately met from stock) by determining the lowest value of $S$ that satisfies the inequality

$$
\sum_{k=0}^{S-1} \frac{(? n L)^{k} e^{-? n L}}{k !} \geq p
$$

where the left-hand side expresses the probability that the demand during the replenishment lead-time will not exceed $S$-1, the available stock just after one part has been retrieved from stock and a replenishment order has been issued.

The problem with the current method, as described above, is that it takes the parameter ? as known and constant. This is not always justifiable. Especially when the company wants to introduce a new type of circuit pack to the market, it is obvious that it has no real data over the failure rate. There exists only an initial failure estimate, which is used as the parameter? and is here denoted ? (KT uses its own terms for the estimates of ?, which are purposefully disguised). This estimate is obtained from a reliability prediction method used during the design of the equipment, in this case the parts count method ${ }^{3}$, which counts the number of parts in the equipment / module and adds their failure rates up according to their number (assuming every part is in a reliability series configuration).

As soon as the company receives the first real data for an item, after a twelve-month installation period, it proceeds with the computation of $?_{1}$, which is the observed real failure rate of installed circuit packs, computed from

$$
?_{1}=r / n t
$$

$r$ is the number of observed failures during the operational time $t$, which is usually equal to one year (8760 hours). $?_{1}$ is calculated separately for each location and the $?_{1} / ?_{0}$ ratio is used as an indication of prediction error. However, although ? ${ }_{1}$ changes with time as new data is collected, it is not used directly as the Poisson parameter ? in (1), because of the fear that the real failure rate may thus be underestimated. Instead, $?_{2}$ is eventually calculated as the upper $95 \%$ confidence limit of ? ${ }_{1}$, using the fact that the confidence limits on the mean of a Poisson distribution are derived by means of the chi-square distribution ${ }^{4}$ with $2 r+2$ degrees of freedom. The exact formula for calculating $?_{2}$ is

$$
?_{2}=\frac{X^{2}(0.95,2 r+2)}{2 n t}
$$




\section{Example}

$n=4010$ circuit packs of a certain type were installed at a particular location. After one year of operation ( $t=8760$ hours) $r=171$ failures had occurred. The replenishment lead-time was $L=1428$ hours and the target service level $95 \%(p=0.95)$. It turns out that $?_{1}=4.868 \cdot 10^{-6}$ failures per hour and $?_{2}=5.526 \cdot 10^{-6}$ failures per hour. Using (1) with $?=?_{2}$, the minimum base stock that is required is $S=42$ spare parts, providing a service level of $95.5 \%$.

\section{General framework of the Bayesian approach}

Although the current method is certainly reasonable, two important issues arise:

a) By assuming that the parameter ? is constant, the uncertainty that characterises the average failure rate is not taken explicitly into account.

b) By using the upper $95 \%$ confidence limit of the observed failure rate as the estimate of ?, it is possible that the method, in an effort to account for the fluctuation of the real failure rate, may be unduly conservative and may thus result in higher stock than necessary.

These concerns may be addressed using a Bayesian approach for the estimation of the demand for spare parts. Specifically, the uncertainty about ? may be treated by assigning to it a prior probability distribution, to be updated as a posterior distribution on the basis of new observations (failures). The appropriate and convenient distribution of ? is the conjugate prior for the Poisson distribution, namely the Gamma distribution with parameters $a, \beta$ and density function

$$
G(? \mid a, \beta)=\frac{\beta^{a} ?^{a-1} e^{-? \beta}}{G(a)}
$$

where $a>0, \beta>0$ and $G(a)$ is the Gamma function. The Gamma distribution has mean $a / \beta$, variance $a / \beta^{2}$ and it is very flexible, as it may have a shape that is either one-tailed $(a \leq 1)$ or two-tailed $(a>1)$. It is well known ${ }^{5}$ that if $r$ demands are observed in a time period of length $t$, then the posterior density function of ? is $G\left(? \mid a^{\prime}, \beta^{\prime}\right)$, with $a^{\prime}=a+r$ and $\beta^{\prime}=\beta+t$. The compound Gamma-Poisson probability function for the number of failures $k$ (demand for spare parts) during the replenishment lead-time $L$ is 


$$
p(k \mid a, \beta)=\int_{0}^{\infty} \frac{(? n L)^{k} e^{-? n L}}{k !} \frac{\beta^{a} ?^{a-1} e^{-? \beta}}{G(a)} d ? .
$$

For convenience and consistency with the measures used by the company, in our application the failure rate, ?, is defined as failures occurred in 1000 installed circuit packs per year. Then, the base stock that provides at least the required service level $p$, which will be termed "critical stock level", is the lowest value of $S$ that satisfies the inequality

$$
\sum_{k=0}^{S-1} \int_{0}^{\infty} \frac{(? n L / 1000)^{k} e^{-? n L / 1000}}{k !} \frac{\beta^{a} ?^{a-1} e^{-? \beta}}{G(a)} d ? \geq p
$$

To apply the method, initial values of the unknown parameters $a, \beta$ of the prior Gamma distribution have to be specified. This is a critical and subjective part of the Bayesian approach, because two equations are needed to define $a$ and $\beta$ and these equations can be specified in several alternative ways. A typical approach is to estimate

a) the mean or mode of the distribution of ? (first equation) and

b) a percentile of the distribution of ? (second equation)

by some means, either expert elicitation or some formal, statistical method. For example, the first equation may be obtained by setting the mean of the prior Gamma equal to the original estimate of the failure rate

$$
a / \beta=?_{0},
$$

while the second equation may come from experts' experience, e.g., in $95 \%$ of the cases the actual failure rate does not exceed the double of the originally estimated failure rate ? ${ }_{0}$ :

$$
P\left(? \leq 2 ?_{0}\right)=0.95 \Rightarrow \int_{0}^{2 ?_{0}} \frac{\beta^{a} ?^{a-1} e^{-? \beta}}{G(a)} d ?=0.95 .
$$

After the observation of $r$ failures in $t$ years, the updating of the parameters is done through

$$
\begin{gathered}
a^{\prime}=a+r, \\
\beta^{\prime}=\beta+t n / 1000 .
\end{gathered}
$$

Since? is expressed per 1000 units per year, the interpretation of the updating relationships after $r$ failures in $n$ installed circuit packs in $t$ years is that equivalently $r$ failures are observed in 1000 circuit packs in $t n / 1000$ years.

It is clear that different initial assumptions may be made and consequently expressions different from (7) and (8) may result, leading in turn to different $a, \beta$ values. To explore the effect of these assumptions we examine below, through an example, four alternatives, which result from a combination of two decisions. The first concerns the choice 
to set either the mean, $a / \beta$, or the mode, $(a-1) / \beta$, of the Gamma distribution equal to ?. The dilemma is due to the usual difficulty to characterise the single-valued estimate of the failure rate as an average or a most probable value. The second decision concerns the upper limit of the integral in (8). In the case of KT's circuit packs, some of the company experts expressed the opinion that it should be set equal to $1.5 ?_{0}$ rather than $2 ?_{0}$. To summarise, the four alternatives use the following expressions for determining $a, \beta$ of the initial prior:

First alternative: $\quad a / \beta=?_{0} \quad$ and $\quad \int_{0}^{2} \frac{\beta^{a} ?^{a-1} e^{-? \beta}}{G(a)} d ?=0.95$

Second alternative: $a / \beta=?_{0} \quad$ and $\quad \int_{0}^{1.5} \frac{?_{0}^{0}}{G(a)} d ?=0.95$.

Third alternative: $\quad(a-1) / \beta=?_{0} \quad$ and $\quad \int_{0}^{2 ?_{0}} \frac{\beta^{a} ?^{a-1} e^{-? \beta}}{G(a)} d ?=0.95$

Fourth alternative: $(a-1) / \beta=?_{0} \quad$ and $\quad \int_{0}^{1.5} \frac{?^{2}}{G(a)} d ?=0.95$.

\section{Example}

Let us reconsider the example of the previous section $(n=4010$ installed circuit packs, $r=171$ failures in $t=1$ year, $L=0.163$ years, $p=0.95$ ). The original estimate of the failure rate was $?_{0}=81.5$ failures per 1000 units per year and the observed failure rate after one year is $?_{1}=42.64$ failures per 1000 units per year.

For each of the four alternative rules, Table 1 shows the resulting $a, \beta$, the mean $\mu_{L}$ and the standard deviation $s_{L}$ of the compound Gamma-Poisson distribution for the demand (failures) during $L$, as well as the critical stock level $S$. The system of the two equations for $a$ and $\beta$ was solved approximately with the requirement that $a$ is always a positive integer, so that the Gamma function is easily evaluated: $G(a)=(a-1)$ !. The values of $\mu_{L}$ and $s_{L}$ refer to the number $n=4010$ of installed units. It is clear from Table 1 that the choice of the mean or the mode does not affect significantly the outcome, in contrast to the choice of the upper $95 \%$ limit on ? Responsible for the noticeably larger critical stock level with the first and third rules is apparently the much larger variance of the demand during the replenishment leadtime $L$. 


\section{Table 1 about here}

When the initial priors are updated using the data on failures after one year $(r=171)$, the new parameters $a^{\prime}, \beta^{\prime}$ are also shown in Table 1 , together with the new $\mu_{L}{ }^{\prime}, s_{L}{ }^{\prime}$ and $S^{\prime}$. It is easily seen that almost independently of the rule used for determining the initial $a, \beta$, the updated critical stock level $S^{\prime}$ is more or less the same and much lower than $S$. In other words, the end result is not sensitive to the choice of the initial prior. This is a very encouraging finding, because it suggests that the effect of the only arbitrary part of the Bayesian approach is diminishing quickly. However, if the prior has a very small variance, the posterior has a strong "memory", i.e., it is affected more by the prior. This would be the case for a circuit pack that has been in operation for a relatively long time and the prior would then be formed based on a large number of observed failures. Since that prior is no more an initial prior but a comprehensive summary of available information, this type of insensitivity in updating is definitely an additional appealing feature of the Bayesian approach.

It is also worth noting that the minimum base stock that is required according to the Bayesian method is $S^{\prime}=39$ (rules $1,2,3$ ) or $S^{\prime}=40$ spare parts (rule 4), while the appropriate critical stock level according to the current method is $S^{\prime}=42$. The explanation for the difference between the two methods is that the demand forecasted using the Bayesian approach is lower than the one forecasted by the current KT method. Figure 1 shows the Gamma-Poisson demand distributions as they come out by the four Bayesian alternatives (which they almost coincide), along with the Poisson demand distribution as it comes out by the company's current method.

\section{Figure 1 about here}

\section{Application and results}

The preceding section exhibited and explained the general framework of the Bayesian approach, making frequent reference to a specific example. The exposition reveals some problematic issues with respect to practical application of the method in the case under consideration:

a) How accurate is the original estimate of the failure rate ?? Although the effect of the initial prior is quickly diminishing, a largely inaccurate estimate ?0 may lead to extremely erroneous decisions for new circuit packs, with no failure data available. 
b) How reliable are the subjective estimates of upper percentiles? The experts tend to provide such estimates without indicating or being able to specify that they refer to the 90 or 95 or some other percentile.

To resolve these issues we proceeded by first considering a generalisation of the four alternative rules examined in the previous section. Specifically, for a circuit type with initial estimate of the failure rate ? (obtained by the parts count method described in Section 2), the following system of equations is formulated:

$$
\begin{gathered}
a / \beta=? ?_{0} \\
P\left(? \geq_{0} ?_{0}\right)=0.95 \Rightarrow \int_{0}^{d ?_{0}} \frac{\beta^{a} ?^{a-1} e^{-? \beta}}{G(a)} d ?=0.95
\end{gathered}
$$

where the values ? and $d$ are to be evaluated. In order to specify these values for our case, we made a comparison between the initial estimates of $?_{0}$ and the actually observed failure rates $?_{1}$ for all circuit packs for which real failure data was available. The idea is to obtain an indication of how far $\lambda_{0}$ may be from the actual failure rate $\lambda_{1}$ and then use this spread to estimate a general prior which is scaled to the value of $\lambda_{0}$, using equation (11) and (12), over all circuit packs and locations. More specifically, ? and $d$ are first evaluated for all circuit packs with a history. Then, their averages are computed and finally these average values are used in (11) and (12) to estimate $a$ and $\beta$ for new circuit packs, in conjunction with the estimates of their respective $\lambda_{0}$.

The procedure for evaluating ? and $d$ is illustrated below for circuit pack A, which at the end of 1997 had already been installed for four years (1994-1997) in 12 locations. Table 2 contains the failure data for that circuit pack at the 12 locations, including the data for 1998, which was used later for updating $a, \beta$.

Table 2 about here

The observed failure rate $?_{1}$ and then the ratio $?_{1} / ?_{0}$ is computed for all 12 locations and four years. The $?_{1} / ?_{0}$ ratios are given in Table 3 . The $?_{1} / ?_{0}$ values of locations 8,9 and 11 , where less than 100 units are installed, are considered unreliable and are therefore excluded from the calculations that follow. (It is seen from Table 3 that some of these values at locations 8 and 11, where only 32 and 24 units are installed, are equal to zero, therefore misleading). We now assume that all remaining $9.4=36 ?_{1} / ?_{0}$ ratios are independent observations of the natural spread of the actual failure rate around the predicted failure rate, caused by differences in location, product and use aspects. The average of those ratios is 0.5 
and ? is set equal to this value. It is clear from (11) that this procedure amounts to set the mean $a / \beta$ of the Gamma prior equal to the average observed failure rate? .

\section{Table 3 about here}

Since $95 \%$ of 36 (total number of usable $?_{1} / ?_{0}$ ratios) is 34.2 , the value $?_{1} / ?_{0}$ under which $95 \%$ of the observations lie is the $34^{\text {th }}$ (or third largest) $?_{1} / ?_{0}$ ratio. In this case, this value is 0.7 (after excluding the two largest values, namely 0.83 and 0.74 ) and consequently $d$ $=0.7$. Using of ? $=0.5$ and $d=0.7$ in (11) and (12) yields $a=25.5$ and $\beta=0.61$. Then, the critical stock levels $S$ for circuit packs A in 1998 result from (6) with the appropriate $n$ and $L$ $=0.163$ years at each location. The leftmost columns of Table 4 contain the critical $S$ in 1998 and the resulting service levels $p$ at the 12 locations.

\section{Table 4 about here}

The above procedure is repeated for all circuit packs with a failure history. The average value of ? is 0.42 (meaning that ? $?_{0}$ is generally a rather pessimistic estimate of the real failure rate, possibly because of the conservatism of the series configuration assumption in estimating ? $?_{0}$ ) and the average value of $d$ is 1.46 . For new circuit packs with no available observations, these averages are used as approximate values of ? and $d$ in (11) and (12) respectively to obtain the parameters $a, \beta$ of the initial prior distribution.

This method to determine the prior seems to be new; at least we have not seen similar applications of Bayesian techniques to forecast demand and determine the appropriate parameters of inventory systems like the one under consideration.

After obtaining new data ( $r$ failures in 1998), the parameters $a, \beta$ are updated according to (9) and (10). At location 1, for example, $a^{\prime}=a+r=25.5+95=120.5$ and $\beta^{\prime}=$ $\beta+t n / 1000=0.61+1871 / 1000=2.48$. Table 4 shows the resulting critical $S$ in 1999 and corresponding service levels $p$ at the 12 locations. The table also includes the same information for 1998 and 1999 using the company's current method of computing the critical stock levels. For 1998, the initial estimate ?0 was used as the failure rate, whereas for 1999, the current method was applied with ? $?_{2}$ computed from (3), taking into account the $r$ observed failures in 1998. It is obvious that the number of spare parts that should be kept in inventory to provide the required customer service is significantly lower under the proposed method.

The comparison in Table 4 is somewhat uneven, because in contrast to the proposed method the current KT method used less data, namely, it did not take advantage of the failure data of years 1994-1997. A more meaningful comparison, where both methods use the same data, is conducted by means of Table 5, which shows the critical stock levels to achieve at least a 95\% service level for selected circuit packs (including type A), differing in the 
initially estimated ? , at various locations. The table shows the installed circuit packs at the locations, $n$, the observed failures $r(T)$ in a certain year $T$ and the critical stock levels $S_{T}$ and $S_{T+1}$ by the current and the proposed method for years $T$ and $T+1$ respectively. The procedure of computing $S_{T}, S_{T+1}$ under the current method is identical to the procedure used for constructing Table 4. However, the calculation of $S_{T}$ for year $T$ under the proposed Bayesian method is done as for the case of new circuit packs (without using any failure information other than ? ${ }_{0}$ ), i.e., it is based on computing $a$ and $\beta$ from (11) and (12) with ? $=0.42$ and $d=$ 1.46. Then, $S_{T+1}$ is computed by updating $a, \beta$, using $r(T)$.

\section{Table 5 about here}

The general conclusion from Table 5 is that the proposed method recommends significantly lower stock levels of spare parts for the installed circuit packs. The reduction in total stock for all three circuit pack types is $11.4 \%$ for year $T$ (initial estimates and computations without any failure observations) and $13.5 \%$ for year $T+1$ (after accumulation and consideration of real failure data). For year $T$ the reduction is certainly expected because the current method uses ? as the failure rate, while the proposed method uses $0.42 ?_{0}$ as the average failure rate. For year $T+1$, the stock reduction may again be attributed partly to the different initial estimates of the failure rate, but it is even higher than in year $T$, thus proving that the estimate $?_{2}$ is rather conservative. Comparing the stock reduction for different types of circuit packs, one observes that for type A, which has the highest initial estimate of the failure rate $\left(?_{0}=81.5\right.$ failures per 1000 units per year), the reduction is $8.9 \%(T)$ to $10.9 \%$ $(T+1)$; for type B with $?_{0}=68.3$ and low usage $(n)$, the reduction is $19 \%(T)$ to $20 \%(T+1)$ and for type $\mathrm{C}$ with $?_{0}=6.1$ and high usage the stock reduction is $12.7 \%(T)$ to $14.1 \%(T+1)$. Thus, no systematic relationship between either $?_{0}$ or $n$ and stock reduction is identified.

A final comment concerns the calculation of stock of type A at year $T$, under the proposed Bayesian approach. When the critical stock levels at the 12 locations were computed for year $1998(T)$ taking into account the available data from 1994 to 1997, the resulting total stock (Table 4) was only 224 spare parts. When these stock levels were computed ignoring the previous failure data, the total stock (Table 5) was much higher, namely 326 spare parts. However, in both cases the total stock for the following year (using of course the same observations from year $T$ ) is exactly the same, 204 units. The difference at year $T$ is explained by the much larger $d$ in the second case (1.46 as opposed to 0.7 in the first case) despite the smaller ? (0.42 in the second case compared to ? $=0.5$ in the first case). The lack of difference at year $T+1$ confirms the robustness of the Bayesian method to the parameters of the initial prior Gamma distribution of ?. 


\section{Conclusion}

In this paper we presented a case study of applying a Bayesian approach to forecasting the demand for spare parts of electronic equipment, with the objective of more accurate determination of stock levels required to provide a negotiated service level to the users of the equipment. The proposed method is not much more complicated than the one currently in use by the company manufacturing and providing the spare parts. Using the same form of inventory control policy, i.e., the $(S-1, S)$ system, the Bayesian method results in lower total stock for the same level of service.

The method presented in this paper works under the assumption that the failure data originate from a stationary process (apart from the number of units installed in the field, for which an easy correction can be made). One should always verify this assumption. In one case we had data indicating a tenfold lower failure rate than the prior. In the Bayesian updating the posterior got a mean in between the mean of the prior and the average of the data and the variance of the posterior was lower than that of the prior, because of the additivity in the updating formulas (9) and (10). This result, however, was surprising and counterintuitive (as we expected the variance to increase), yet a consequence of the stationarity assumption. Later on, the data appeared to contain errors and the correct data was in line with the prior. A similar issue is a possible ageing of the units. If that occurs one might regard old data as less important than new data and do not add the number of observations, but consider weighting the old values with a forgetting factor.

The proposed method has been received very well by the company. As we also advised them to use a METRIC like method for their multi-echelon inventory system, the implementation of the Bayesian method was delayed to a later stage.

\section{Acknowledgement}

The research presented in this paper has been supported by grants from the European Union in the Erasmus and TMR programs (project Reverse Logistics ERB 4061 PL 97-5650). The authors would like to thank T. van der Ploeg from KT for his support during the study. 


\section{References}

1. Kaplan, A.J. Bayesian approach to inventory control of new parts, IIE Transactions 20, 151-156, 1988.

2. Sherbrooke, C.G. Optimal inventory modelling of systems, John Wiley and Sons, New York 1992.

3. MIL-HDBK-217F, Military Handbook, Reliability prediction of electronic equipment, Department of Defense, Washington DC, 20301.

4. Kapur, K.C. and Lamberson, L.R. Reliability Engineering Design, John Wiley and Sons, New York 1977.

5. Carlin, B.P. and Louis, T.A. Bayes and Empirical Bayes Methods for Data Analysis, Chapman and Hall, London 1996. 
Table 1: Sensitivity analysis on the choice of initial prior distribution for the failure rate

\begin{tabular}{ccccccccccc}
\hline Rule & $a$ & $\beta$ & $\mu_{L}$ & $s_{L}$ & $S$ & $a^{\prime}$ & $\beta^{\prime}$ & $\mu_{L}^{\prime}$ & $s_{L}^{\prime}$ & $S^{\prime}$ \\
\hline 1 & 4 & 0.049 & 53.2 & 27.6 & 106 & 175 & 4.059 & 28.2 & 5.7 & 39 \\
2 & 13 & 0.159 & 53.2 & 16.5 & 84 & 184 & 4.169 & 28.8 & 5.8 & 39 \\
& & & & & & & & & & \\
3 & 7 & 0.074 & 62.1 & 24.8 & 108 & 178 & 4.084 & 28.5 & 5.8 & 39 \\
4 & 18 & 0.209 & 56.4 & 15.3 & 84 & 189 & 4.219 & 29.3 & 5.8 & 40 \\
\hline
\end{tabular}


Table 2: Observed failures of circuit pack A and installed basis at the 12 locations

\begin{tabular}{ccccccc}
\hline Location & 1994 & 1995 & 1996 & 1997 & 1998 & Units $(n)$ \\
\hline 1 & 70 & 100 & 92 & 106 & 95 & 1871 \\
2 & 146 & 176 & 180 & 161 & 188 & 4008 \\
3 & 55 & 65 & 53 & 61 & 55 & 1071 \\
4 & 106 & 21 & 132 & 171 & 112 & 3784 \\
5 & 15 & 23 & 13 & 23 & 16 & 1016 \\
6 & 29 & 48 & 64 & 88 & 41 & 1616 \\
7 & 51 & 37 & 28 & 36 & 43 & 752 \\
8 & 0 & 0 & 1 & 1 & 0 & 32 \\
9 & 2 & 3 & 6 & 5 & 1 & 64 \\
10 & 163 & 202 & 143 & 177 & 140 & 3792 \\
11 & 0 & 0 & 2 & 0 & 0 & 24 \\
12 & 74 & 78 & 101 & 79 & 90 & 1840 \\
\hline
\end{tabular}

Table 3: Values of $?_{1} / ?_{0}$ ratios for circuit pack A at the 12 locations

\begin{tabular}{cccccc}
\hline Location & 1994 & 1995 & 1996 & 1997 & 1998 \\
\hline 1 & 0.46 & 0.66 & 0.60 & 0.70 & 0.62 \\
2 & 0.45 & 0.54 & 0.55 & 0.49 & 0.58 \\
\hline
\end{tabular}




\begin{tabular}{cccccc}
\hline 3 & 0.63 & 0.74 & 0.61 & 0.70 & 0.63 \\
4 & 0.34 & 0.07 & 0.43 & 0.55 & 0.36 \\
5 & 0.18 & 0.28 & 0.16 & 0.28 & 0.19 \\
6 & 0.22 & 0.36 & 0.49 & 0.67 & 0.31 \\
7 & 0.83 & 0.60 & 0.46 & 0.59 & 0.70 \\
8 & 0.00 & 0.00 & 0.38 & 0.38 & 0.00 \\
9 & 0.38 & 0.58 & 1.15 & 0.96 & 0.19 \\
10 & 0.53 & 0.65 & 0.46 & 0.57 & 0.45 \\
11 & 0.00 & 0.00 & 1.02 & 0.00 & 0.00 \\
12 & 0.49 & 0.52 & 0.67 & 0.53 & 0.60 \\
\hline
\end{tabular}

Table 4: Critical stock and service level for circuit pack A under the proposed and current method

\begin{tabular}{c|cc|cc|cc|cc}
\hline \multirow{2}{*}{ Location } & \multicolumn{4}{|c|}{ Proposed method } & \multicolumn{3}{c}{ Current method } \\
\cline { 2 - 9 } & \multicolumn{2}{|c|}{1998} & \multicolumn{2}{|c|}{1999} & \multicolumn{2}{|c|}{1998} & \multicolumn{2}{c}{1999} \\
\hline 1 & 21 & 95.0 & 23 & 96.3 & 34 & 95.3 & 26 & 94.6 \\
2 & 41 & 95.0 & 41 & 95.4 & 66 & 95.0 & 45 & 95.0 \\
3 & 14 & 96.7 & 15 & 96.9 & 22 & 96.7 & 18 & 96.2 \\
4 & 39 & 95.0 & 28 & 95.3 & 63 & 95.4 & 30 & 95.5 \\
5 & 13 & 95.8 & 9 & 96.4 & 21 & 96.5 & 8 & 95.1 \\
\hline
\end{tabular}




\begin{tabular}{c|cc|cc|cc|cc}
\hline 6 & 19 & 95.9 & 14 & 96.1 & 30 & 95.3 & 15 & 96.8 \\
7 & 10 & 95.0 & 12 & 97.0 & 16 & 95.2 & 15 & 95.7 \\
8 & 2 & 97.9 & 2 & 98.1 & 3 & 99.1 & 3 & 99.1 \\
9 & 3 & 98.9 & 3 & 99.1 & 4 & 98.9 & 4 & 98.9 \\
10 & 39 & 95.0 & 33 & 95.7 & 63 & 95.3 & 36 & 95.9 \\
11 & 2 & 98.8 & 2 & 98.8 & 2 & 95.9 & 3 & 99.6 \\
12 & 21 & 95.6 & 22 & 96.0 & 34 & 96.1 & 26 & 96.6 \\
\hline Total & 224 & 96.2 & 204 & 96.8 & 358 & 96.2 & 229 & 96.6 \\
\hline
\end{tabular}


Table 5: Critical stock levels for circuit pack types A $\left(?_{0}=81.5\right), \mathrm{B}\left(?_{0}=68.3\right), \mathrm{C}\left(?_{0}=6.1\right)$ under the proposed and current method

\begin{tabular}{|c|c|c|c|c|c|c|c|c|c|c|c|c|c|c|c|c|c|c|}
\hline \multirow[b]{3}{*}{ Loc. } & \multicolumn{6}{|c|}{ Circuit pack type A } & \multicolumn{6}{|c|}{ Circuit pack type B } & \multicolumn{6}{|c|}{ Circuit pack type $\mathrm{C}$} \\
\hline & \multirow[b]{2}{*}{$n$} & \multirow[b]{2}{*}{$r(T)$} & \multicolumn{2}{|c|}{ Proposed } & \multicolumn{2}{|c|}{ Current } & \multirow[b]{2}{*}{$n$} & \multirow[b]{2}{*}{$r(T)$} & \multicolumn{2}{|c|}{ Proposed } & \multicolumn{2}{|c|}{ Current } & \multirow[b]{2}{*}{$n$} & \multirow[b]{2}{*}{$r(T)$} & \multicolumn{2}{|c|}{ Proposed } & \multicolumn{2}{|c|}{ Current } \\
\hline & & & $S_{T}$ & $S_{T+1}$ & $S_{T}$ & $S_{T+1}$ & & & $S_{T}$ & $S_{T+1}$ & $S_{T}$ & $S_{T+1}$ & & & $S_{T}$ & $S_{T+1}$ & $S_{T}$ & $S_{T+1}$ \\
\hline 1 & 1871 & 95 & 31 & 24 & 34 & 26 & 184 & 3 & 5 & 4 & 6 & 6 & 3584 & 15 & 6 & 6 & 8 & 8 \\
\hline 2 & 4008 & 188 & 62 & 42 & 66 & 45 & 825 & 22 & 13 & 9 & 15 & 10 & 1920 & 0 & 4 & 3 & 5 & 5 \\
\hline 4 & 3784 & 112 & 59 & 27 & 63 & 30 & 779 & 25 & 12 & 9 & 15 & 11 & 7678 & 19 & 11 & 8 & 13 & 9 \\
\hline 5 & 1016 & 16 & 18 & 7 & 21 & 8 & - & - & - & - & - & - & 20992 & 186 & 26 & 40 & 30 & 45 \\
\hline 6 & 1616 & 41 & 27 & 13 & 30 & 15 & 81 & 1 & 3 & 3 & 4 & 5 & 13695 & 42 & 18 & 13 & 21 & 15 \\
\hline 7 & 752 & 43 & 14 & 13 & 16 & 15 & 464 & 28 & 8 & 9 & 10 & 12 & 31037 & 206 & 37 & 44 & 41 & 49 \\
\hline 10 & 3792 & 140 & 59 & 33 & 63 & 36 & 738 & 29 & 12 & 10 & 14 & 12 & 30134 & 55 & 36 & 16 & 40 & 18 \\
\hline 11 & 24 & 0 & 2 & 2 & 2 & 3 & - & - & - & - & - & - & 1024 & 1 & 3 & 3 & 4 & 4 \\
\hline 12 & 1840 & 90 & 30 & 23 & 34 & 26 & 453 & 25 & 8 & 9 & 11 & 11 & 29210 & 114 & 35 & 27 & 39 & 31 \\
\hline Total & 19870 & 787 & 326 & 204 & 358 & 229 & 3538 & 133 & 64 & 56 & 79 & 70 & 148668 & 677 & 193 & 176 & 221 & 205 \\
\hline
\end{tabular}

